\title{
On the Numerical Solution of Sturm-Liouville Differential Equations
}

1. Introduction. It is the purpose of this paper to use the well-known relation that exists between a Sturm-Liouville differential equation together with its boundary conditions and normalization condition and a problem in the calculus of variations, to obtain an algebraic characteristic value problem from whose solution we may obtain approximations to the characteristic values and characteristic functions of the Sturm-Liouville equation. That is, we shall obtain approximations to the values of $\lambda$ and the functions $y(x)$ satisfying the equation

$$
y^{\prime \prime}+(q(x)+\lambda r(x)) y=0,
$$

such that the boundary conditions

$$
\begin{array}{r}
\alpha_{1} y(a)+\alpha_{2} y^{\prime}(a)=0 \\
\beta_{1} y(b)+\beta_{2} y^{\prime}(b)=0
\end{array}
$$

and the normalization condition

$$
\int_{a}^{b} r y^{2} d x=1
$$

are satisfied, where the prime denotes the derivative of $y(x)$ with respect to $x$, $q$ and $r$ being functions of $x$, and the latter function is required to be positive over the interval

$$
a \leq x \leq b
$$

Equations of the form

$$
\left(p y^{\prime}\right)^{\prime}+q y+\lambda r y=0
$$

may be reduced to that of the form of equation (1.1) by a transformation of the independent variable.

The equations (1.1) and (1.2) themselves may be approximated by a set of linear algebraic equations as follows (cf., Hildebrand [1]). Let us use a set of discrete values of $x$

$$
\begin{aligned}
x_{i} & =a+i h \quad(i=0,1, \cdots, N+1) \\
h & =\frac{b-a}{N+1}
\end{aligned}
$$

and define

$$
y_{i}=y\left(x_{i}\right) \text {. }
$$


Then we may approximate $y^{\prime}\left(x_{i}\right)=y_{i}{ }^{\prime}$ and $-y^{\prime \prime}\left(x_{i}\right)=y_{i}{ }^{\prime \prime}$ by means of finite differences of the $y_{i}$. We would then obtain a set of equations of the form

$$
L_{i j} y_{j}=\lambda r_{i} y_{i}
$$

for the unknowns $\lambda$ and $y_{i}$. That is, we would thus reduce the system of equations (1.1) and (1.2) to an algebraic characteristic value problem. However the matrix $L_{i j}$ would not in general be symmetric. Indeed as the order of the differences used to replace the derivatives increases, the asymmetry of the matrix $L_{i j}$ increases. This asymmetry occurs because the same type of difference formula cannot be used at the ends of the interval $(a, b)$ as in the intericr of the interval.

If the matrix $L_{i j}$ is not symmetric, the reality of the values of $\lambda$ cannot be guaranteed. Moreover the determination of the values of $\lambda$ such that equation (1.4) has a solution is a much greater computational problem when $L_{i j}$ is an asymmetric matrix than when it is a symmetric one. The methods that will be used in the sequel to replace the system of equations (1.1) to (1.3) by an algebraic characteristic value problem will always lead to symmetric matrices.

2. The Variational Problem. It is well known that equation (1.1) is the Euler condition that the integral

$$
\int_{a}^{b}\left(\left(y^{\prime}\right)^{2}-q y^{2}\right) d x
$$

be an extremal where the functions $y(x)$ satisfy equation (1.3).

Hence the system of equations (1.1) to (1.3) is equivalent to finding functions $y(x)$ subject to equations (1.2) such that the integral

$$
I(y)=\int_{a}^{b}\left[\left(y^{\prime}\right)^{2}-q y^{2}\right] d x-\lambda \int_{a}^{b} r y^{2} d x
$$

is an extremum where $\lambda$ is a Lagrange multiplier and the functions $y(x)$ are restricted to satisfy the conditions (1.2).

Instead of dealing with the differential equation (1.1) we deal with the integral (2.2). There are two methods that we shall consider: (1) approximating this integral by using quadrature and finite difference formulas (Method I), and (2) restricting the class of admissible functions to be made up of polynomial arcs (Method II). The latter method is of course a variant of the Rayleigh-Ritz method. However it differs from that method in that it uses a set of polynomials, each of degree $m$, say, and each one defined over an interval $x_{i} \leq x<x_{i+1}$, to represent an admissible function $y(x)$ instead of an expansion of $y(x)$ in terms of a convenient orthonormal set of functions. The latter representation is required to hold over the entire interval $(a, b)$ and many terms may be needed because of the behavior of $y(x)$ on a few of the intervals $x_{i} \leq x<x_{i+1}$; whereas in the former representation we may use intervals of varying sizes or allow the admissible function to be made up of polynomial arcs, where the degree of each arc need not be the same.

3. Method I. In the discussion of this method we shall restrict ourselves to the case where $\alpha_{2}=\beta_{2}=0$ in equations (1.2). The discussion may be readily modified to take care of more general boundary conditions. 
The first step is to obtain a finite difference approximation to (2.2). To do this we introduce the quadrature formula

$$
\int_{a}^{b} \phi(x) d x=\sum_{i=0}^{N+1} w_{i} \phi_{i}+\epsilon_{1},
$$

where $\epsilon_{1}$ is the error in this formula. The weighting factors $w_{i}$ for the functional values $\phi_{i}$ are to be determined later. We also introduce the formula

$$
y_{i}^{\prime}=h^{-1} \sum_{j=0}^{N+1} a_{i j} y_{j}+\eta_{i} \quad(i=0,1, \cdots, N+1)
$$

which is a finite difference approximation to the first derivative at $x_{i}$ in terms of the functional values $y_{i}$. The values of $a_{i j}$ are to be determined later also.

Using (3.1) and (3.2) we approximate the first integral in (2.2) obtaining

$$
\begin{aligned}
\int_{a}^{b}\left[\left(y^{\prime}\right)^{2}-q y^{2}\right] d x & =\sum_{i=0}^{N+1} w_{i}\left[\left(y_{i}{ }^{\prime}\right)^{2}-q_{i} y_{i}{ }^{2}\right]+\epsilon_{1} \\
& =\sum_{i=0}^{N+1} w_{i}\left[h^{-1} \sum_{j=0}^{N+1} a_{i j} y_{j}+\eta_{i}\right]^{2}-\sum_{i=0}^{N+1} w_{i} q_{i} y_{i}{ }^{2}+\epsilon_{1} \\
& =\sum_{j, k=0}^{N+1}\left[h^{-2} \sum_{i=0}^{N+1} w_{i} a_{i j} a_{i k}-w_{j} q_{j} \delta_{j k}\right] y_{j} y_{k}+\left(\epsilon_{1}+\epsilon_{2}\right)
\end{aligned}
$$

where

$$
\epsilon_{2}=\sum_{i=0}^{N+1} w_{i}\left[2 \eta_{i} h^{-1} \sum_{j=0}^{N+1} a_{i j} y_{j}+\eta_{i}{ }^{2}\right]
$$

and $\delta_{j k}$ is the Kronecker delta. Our equation becomes

$$
\int_{a}^{b}\left[\left(y^{\prime}\right)^{2}-q y^{2}\right] d x=\sum_{j, k=0}^{N+1} L_{j k} y_{j} y_{k}+\left(\epsilon_{1}+\epsilon_{2}\right)
$$

if we set

$$
L_{j k}=h^{-2} \sum_{i=0}^{N+1} w_{i} a_{i j} a_{i k}-w_{j} q_{j} \delta_{j k}
$$

Likewise the second integral in (2.2) becomes

$$
\int_{a}^{b} r y^{2} d x=\sum_{j, k=0}^{N+1} w_{j} r_{j} \delta_{j k} y_{j} y_{k}+\epsilon_{3} .
$$

Thus $I(y)$ is approximated by the quadratic form

$$
F=\sum_{j, k=0}^{N+1} L_{j k} y_{j} y_{k}-\lambda^{*} \sum_{j, k=0}^{N+1} w_{j} r_{j} \delta_{j k} y_{j} y_{k}
$$


and if we insert the boundary conditions $y_{0}=y_{N+1}=0$ we obtain simply

$$
F=\sum_{j, k=1}^{N} L_{j k} y_{j} y_{k}-\lambda^{*} \sum_{i, k=1}^{N} w_{j} r_{j} \delta_{j k} y_{j} y_{k}
$$

The second step in our numerical method is to minimize the quadratic form. This is a simple problem in the calculus and the necessary condition for a minimum is that

$$
\frac{\partial F}{\partial y_{j}}=\sum_{k=1}^{N} L_{j k} y_{k}-\lambda^{*} \sum_{k=1}^{N} w_{j} r_{j} \delta_{j k} y_{k}=0,
$$

for $j=1, \cdots, N$. This system of linear equations can be written in matrix notation

$$
L y-\lambda^{*} W R y=0,
$$

where $L$ is a symmetric $N \times N$ matrix and $W R$ is a diagonal one.

In order to put (3.7) in a form analogous to (1.1) we multiply through by $-1 / w_{j}$, and if we define $t_{j k}$ to be

we can write

$$
t_{j k}=-\left(1 / w_{j}\right) L_{j k}
$$

$$
\sum_{k=1}^{N} t_{j k} y_{k}+\lambda^{*} r_{j} y_{j}=0
$$

which becomes

$$
T y+\lambda^{*} R y=0,
$$

in matrix form. Using (3.4) and setting

$$
G_{j}=1 / w_{j} h^{2},
$$

we can write $t_{j k}$ as

$$
t_{j k}=-G_{j} \sum_{i=0}^{N+1} w_{i} a_{i j} a_{i k}+q_{j} \delta_{j k}
$$

We note that $L$ and $T$ are functions of $w_{j}$ and $a_{i j}$ from the approximation formulas (3.1) and (3.2) respectively. In the next section a method will be discussed for selecting $w_{j}$ and $a_{i j}$ so as to have $T$ approximate the differential operator

$$
D_{2} \equiv \frac{d^{2}}{d x^{2}}+q
$$

to a specified order of approximation.

4. The Order of Approximation of the Method. It was stated in the last section that the matrix operator $T$ is to be an approximation to the differential operator $D_{2}$. We would like to specify the order of this approximation. However, before doing so let us define what we mean by a certain "order of approximation" in this case. 
In order to compare a differential operator with a matrix operator we need to arrive at a common basis for comparison. To do this consider the vector

$$
v_{1}=\left[\begin{array}{c}
q_{1} y_{1}+y_{1}^{\prime \prime} \\
\cdot \\
\cdot \\
q_{j} y_{j}+y_{j}^{\prime \prime} \\
\cdot \\
\cdot \\
\cdot \\
q_{N} y_{N}+y_{N}^{\prime \prime}
\end{array}\right],
$$

whose components are obtained by evaluating $D_{2} y$ at the discrete set of mesh points $x_{j}, j=1, \cdots, N$. Also consider the vector

$$
v_{2}=\left[\begin{array}{c}
\sum_{k=1}^{N} t_{1 k} y_{k} \\
\cdot \\
\cdot \\
\cdot \\
\sum_{k=1}^{N} t_{j k} y_{k} \\
\cdot \\
\cdot \\
\sum_{k=1}^{N} t_{N k} y_{k}
\end{array}\right]
$$

Definition. If

$$
\sum_{k=1}^{N} t_{j k} y_{k}=q_{j} y_{j}+y_{j}^{\prime \prime}+0\left(h^{\sigma}\right)
$$

for $j=1, \cdots, N$, then the matrix $T$ approximates the differential operator $D_{2}$ to order $\sigma$.

This definition can be used as a basis for the selection of $w_{j}$ and $a_{i j}$ for formulas (3.1) and (3.2) respectively. To see this let us require that $w_{j}$ and $a_{i j}$ be chosen in such a way that the numerical method is of order $\sigma$. Let us further require that

$$
y_{i}^{\prime}=h^{-1} \sum_{k=0}^{N+1} a_{i k} y_{k}+0\left(h^{\sigma}\right)
$$

i.e., that (3.2) be an approximation formula also of order $\sigma$. This means that the $a_{i k}$ must satisfy the equations

$$
\sum_{k=0}^{N+1} a_{i k}(k-i)^{\mu}=\delta_{1 \mu} \quad(\mu=0,1, \cdots, \sigma) .
$$


For, if we expand $y_{k}$ about $y_{i}$, we get

$$
y_{k}=\sum_{\mu=0}^{\infty}(k-i)^{\mu} y_{i}{ }^{(\mu)} \frac{h^{\mu}}{\mu !}
$$

and thus, in (4.2),

$$
h^{-1} \sum_{k=0}^{N+1} a_{i k} y_{k}=\sum_{\mu=0}^{\infty}\left[\sum_{k=0}^{N+1} a_{i k}(k-i)^{\mu}\right] y_{i}{ }^{(\mu)} \frac{h^{\mu-1}}{\mu !}
$$

from which (4.3) follows.

Using the same procedure, and recalling that $y_{0}=y_{N+1}=0$, we can write the left side of equation (4.1) as

$$
\begin{aligned}
\sum_{k=1}^{N} t_{j k} y_{k}=\sum_{0}^{N+1} t_{j k} y_{k}=\left\{y_{j}\left[\sum_{k=0}^{N+1} t_{j k}\right]+y_{j}{ }^{\prime} h\right. & {\left[\sum_{k=0}^{N+1}(k-j) t_{j k}\right] } \\
& \left.+y_{j}^{\prime \prime} \frac{h^{2}}{2 !}\left[\sum_{k=0}^{N+1}(k-j)^{2} t_{j k}\right]+\cdots\right\} .
\end{aligned}
$$

Thus we have from equation (3.8)

$$
\begin{aligned}
\sum_{k=1}^{N} t_{j k} y_{k}=y_{j}\left[\sum_{k=0}^{N+1}\left(-G_{j} \sum_{i=0}^{N+1} w_{i} a_{i j} a_{i k}+q_{j} \delta_{j k}\right)\right] & \\
+y_{j}{ }^{\prime} h & {\left[\sum_{k=0}^{N+1}(k-j)\left(-G_{j} \sum_{i=0}^{N+1} w_{i} a_{i j} a_{i k}+q_{j} \delta_{j k}\right)\right] } \\
& +y_{j}{ }^{\prime \prime} \frac{h^{2}}{2 !}\left[\sum_{k=0}^{N+1}(k-j)^{2}\left(-G_{j} \sum_{i=0}^{N+1} w_{i} a_{i j} a_{i k}+q_{j} \delta_{j k}\right)\right] \\
& +\cdots
\end{aligned}
$$

Now we are in a position to treat (4.1) as an identity in $y_{i}$ and its derivatives by using the last equation. By equating coefficients of $y_{i}$ and its derivatives up to $y^{(\sigma+1)}$, we obtain

$$
\begin{array}{r}
\sum_{k=0}^{N+1}\left(-G_{j} \sum_{i=0}^{N+1} w_{i} a_{i j} a_{i k}+q_{j} \delta_{j k}\right)=q_{j}, \\
\sum_{k=0}^{N+1}(k-j)\left(-G_{j} \sum_{i=0}^{N+1} w_{i} a_{i j} a_{i k}+q_{j} \delta_{j k}\right)=0, \\
\frac{h^{2}}{2} \sum_{k=0}^{N+1}(k-j)^{2}\left(-G_{j} \sum_{i=0}^{N+1} w_{i} a_{i j} a_{i k}+q_{j} \delta_{j k}\right)=1,
\end{array}
$$

and

$$
\sum_{k=0}^{N+1}(k-j)^{r}\left(-G_{j} \cdot \sum_{i=0}^{N+1} w_{j} a_{i j} a_{i k}+q_{j} \delta_{j k}\right)=0
$$


where $\tau=3, \cdots, \sigma+1$. Since

$$
\sum_{k=0}^{N+1} q_{j} \delta_{j k}=q_{j}
$$

and

$$
(k-j) \delta_{j k}=0,
$$

and using the definition of $G_{j}$, these become

$$
\begin{gathered}
\sum_{i=0}^{N+1} w_{i} a_{i j} \sum_{k=0}^{N+1} a_{i k}=0, \\
\sum_{i=0}^{N+1} w_{i} a_{i j} \sum_{k=0}^{N+1} a_{i k}(k-j)=0, \\
\sum_{i=0}^{N+1} w_{i} a_{i j} \sum_{k=0}^{N+1} a_{i k}(k-j)^{2}=-2 w_{j},
\end{gathered}
$$

and

$$
\sum_{i=0}^{N+1} w_{i} a_{i j} \sum_{k=0}^{N+1} a_{i k}(k-j)^{r}=0
$$

where $r=3, \cdots, \sigma+1$.

A further simplification results if we use equation (4.3), i.e., if we make use of the fact that the $a_{i j}$ give us an approximation to the derivative of order $\sigma$. For we can write

$$
\begin{aligned}
& \sum_{k=0}^{N+1} a_{i k}(k-j)^{\mu}=\sum_{k=0}^{N+1} a_{i k}[(k-i)+(i-j)]^{\mu} \\
& \quad=\sum_{k=0}^{N+1} a_{i k}\left[(k-i)^{\mu}+\mu(k-i)^{\mu-1}(i-j)+\cdots+\mu(k-i)(i-j)^{\mu-1}+(i-j)^{\mu}\right] \\
& \quad=\mu(i-j)^{\mu-1} .
\end{aligned}
$$

With this result our equations become

$$
\begin{aligned}
\sum_{i=0}^{N+1} w_{i} a_{i j} & =0, \\
\sum_{i=0}^{N+1} w_{i} a_{i j}(j-i) & =w_{j},
\end{aligned}
$$

and

$$
\sum_{i=0}^{N+1} w_{i} a_{i j}(j-i)^{r}=0 \quad(\tau=2, \cdots, \sigma),
$$


or simply

$$
\sum_{i=0}^{N+1} w_{i} a_{i j}(j-i)^{\tau}=w_{j} \delta_{1 \tau} \quad(\tau=0, \cdots, \sigma) .
$$

In these equations $j=1, \cdots, N$.

Thus if we select $a_{i j}$ and $w_{j}$ satisfying equations (4.3) and (4.4) our method will give an approximation of order $\sigma$.

5. The First Order Approximation. In this case $\sigma=1$ and equations (4.3) and (4.4) become

$$
\begin{aligned}
\sum_{k=0}^{N+1} a_{i k} & =0, \\
\sum_{k=0}^{N+1} a_{i k}(k-i) & =1 \quad(i=0 \cdots N+1),
\end{aligned}
$$

and

$$
\begin{aligned}
\sum_{i=0}^{N+1} w_{i} a_{i j} & =0, \\
\sum_{i=0}^{N+1} w_{i} a_{i j}(j-i) & =w_{j} \quad(j=1, \cdots, N),
\end{aligned}
$$

respectively. Note that equations (5.1) give conditions on the rows of the matrix $\left(a_{i k}\right)$ whereas equations (5.2) give conditions on the columns (except for the first and last) of the same matrix.

It is easily verified that the matrix

$$
\left(a_{i k}\right)=\left[\begin{array}{rrrrrrrrrr}
-1 & 1 & 0 & 0 & 0 & . & & & & \\
0 & -1 & 1 & 0 & 0 & . & & & & \\
0 & 0 & -1 & 1 & 0 & . & & & & \\
0 & 0 & 0 & -1 & 1 & . & & & & \\
. & . & . & . & \cdot & . & . & . & . & . \\
& & & & & . & -1 & 1 & 0 & 0 \\
& & & & & . & 0 & -1 & 1 & 0 \\
& & & & & . & 0 & 0 & -1 & 1 \\
& & & & & . & 0 & 0 & -1 & 1
\end{array}\right] \text {, }
$$

and $w_{i}$ satisfying

$$
\begin{aligned}
w_{N+1} & =0, \\
w_{i} & =w_{i+1} \quad(i=0, \cdots, N-1)
\end{aligned}
$$

constitute a solution of (5.1) and (5.2), i.e., equations (5.3) and (5.4) give us $a_{i k}$ and $w_{i}$ for the first order case.

Equation (5.3) states that we use the simple first order forward difference formula

$$
y_{i}{ }^{\prime}=\left(y_{i+1}-y_{i}\right) / h+\left[y_{i}{ }^{\prime \prime}(h / 2 !)+y_{i}{ }^{(3)}\left(h^{2} / 3 !\right)+\cdots\right]
$$


for $i=0, \cdots, N$, and the simple first order backward difference formula

$$
y_{i}{ }^{\prime}=\left(y_{i}-y_{i-1}\right) / h-\left[y_{i}{ }^{\prime \prime}(h / 2 !)-y_{i}{ }^{(3)}\left(h^{2} / 3 !\right)+\cdots\right],
$$

for $i=N+1$. Equations (5.4) indicate that the quadrature formula to use is the simple first order "Rectangle Rule"

$$
\int_{a}^{b} f(x) d x=w_{1} \sum_{i=0}^{N} f_{i}-\left[f_{1}^{\prime}(h / 2)+\cdots\right] .
$$

Obviously

$$
w_{1}=(b-a) /(N+1)=h,
$$

since (5.5) must hold for $f(x) \equiv 1$.

6. The Second Order Approximation. In this case $\sigma=2$ and equations (4.3) and (4.4) become

$$
\begin{aligned}
\sum_{k=0}^{N+1} a_{i k} & =0, \\
\sum_{k=0}^{N+1} a_{i k}(k-i) & =1, \\
\sum_{k=0}^{N+1} a_{i k}(k-i)^{2} & =0 \quad(i=0, \cdots, N+1),
\end{aligned}
$$

and

$$
\begin{aligned}
& \sum_{i=0}^{N+1} w_{i} a_{i j}=0, \\
& \sum_{i=0}^{N+1} w_{i} a_{i j}(j-i)=w_{j}, \\
& \sum_{i=0}^{N+1} w_{i} a_{i j}(j-i)^{2}=0 \quad(j=1, \cdots, N),
\end{aligned}
$$

respectively.

It has been shown by Gregory [2] that when $N$ is odd a solution to equations (6.1) and (6.2) is given by the matrix $\left(a_{i k}\right)$ of the form

$$
\left(a_{i k}\right)=\left[\begin{array}{cccccccccc}
-3 / 2 & 2 & -1 / 2 & 0 & 0 & 0 & 0 & & & \\
-1 / 2 & 0 & 1 / 2 & 0 & 0 & 0 & 0 & & & \\
1 / 4 & -1 & 0 & 1 & -1 / 4 & 0 & 0 & & & \\
0 & 0 & -1 / 2 & 0 & 1 / 2 & 0 & 0 & & & \\
0 & 0 & 1 / 4 & -1 & 0 & 1 & -1 / 4 & & & \\
\cdot & \cdot & \cdot & \cdot & \cdot & \cdot & \cdot & . & . & . \\
& & & & 0 & -1 / 2 & 0 & 1 / 2 & 0 & 0 \\
& & & & 0 & 1 / 4 & -1 & 0 & 1 & -1 / 4 \\
& & & & 0 & 0 & 0 & -1 / 2 & 0 & 1 / 2 \\
& & & & 0 & 0 & 0 & 1 / 2 & -2 & 3 / 2
\end{array}\right],
$$


and the $w_{i}$ are given by

$$
\begin{aligned}
w_{1} & =4 w_{0}, \\
w_{2} & =2 w_{0}, \\
w_{3} & =4 w_{0}, \\
\cdot & \\
\cdot & \cdot \\
w_{N-1} & =2 w_{0}, \\
w_{N} & =4 w_{0}, \\
w_{N+1} & =w_{0} .
\end{aligned}
$$

If $N$ is even the solutions differ only in the last four rows of the matrix $\left(a_{i k}\right)$ and the last four values of $w_{i}$, i.e.,

$$
\left(a_{i k}\right)=\left[\begin{array}{ccccccccc}
-3 / 2 & 2 & -1 / 2 & 0 & 0 & 0 & & & \\
-1 / 2 & 0 & 1 / 2 & 0 & 0 & 0 & & & \\
1 / 4 & -1 & 0 & 1 & -1 / 4 & 0 & & & \\
\cdot & \cdot & \cdot & \cdot & \cdot & \cdot & . & . & . \\
& & 0 & -1 / 2 & 0 & 1 / 2 & 0 & 0 & 0 \\
& & 0 & 4 / 17 & -16 / 17 & -3 / 34 & 18 / 17 & -9 / 34 & 0 \\
& & 0 & 0 & 0 & -1 / 3 & -1 / 2 & 1 & -1 / 6 \\
& & 0 & 0 & 0 & -1 / 6 & 0 & -1 / 2 & 2 / 3 \\
& & 0 & 0 & 1 / 3 & -1 / 2 & -1 & 7 / 6
\end{array}\right] \text {, }
$$

and

$$
\begin{aligned}
w_{1} & =4 w_{0}, \\
w_{2} & =2 w_{0}, \\
\cdot & \cdot \\
\cdot & =2 w_{0}, \\
w_{N-4} & =4 w_{0}, \\
w_{N-3} & =\frac{17}{8} w_{0}, \\
w_{N-2} & \\
w_{N-1} & =\frac{27}{8} w_{0}, \\
w_{N} & =\frac{27}{8} w_{0}, \\
w_{N+1} & =\frac{9}{8} w_{0} .
\end{aligned}
$$

Equations (6.6) can be interpreted as a quadrature formula consisting of Simpson's Rule for all but the last three intervals and the "3/8 Rule" for the last three intervals. This special treatment at the end is due to the fact that Simpson's Rule can only be used with an odd number of mesh points and here $N$ is even.

In a later section the solutions of equations (3.7), where $L_{i j}$ is defined by equation (3.4) for the first and second order approximations, will be compared 
to the characteristic values and values of the characteristic functions at the mesh points of the Sturm-Liouville system described by the equation

$$
\frac{d^{2} y}{d x^{2}}+\lambda y=0
$$

with the boundary conditions

$$
y(0)=y(1)=0
$$

and the normalization condition

$$
\int y^{2} d x=1
$$

This simple example for which the solutions are known to be

$$
\begin{gathered}
\lambda_{n}=\pi^{2} n^{2} \\
y_{n}=\sqrt{2} \sin \pi n x
\end{gathered}
$$

will be used in the discussion of the effectiveness of Methods I and II.

7. Method II. We now turn our attention to the derivation of the algebraic characteristic value problem associated with Method II. In this method, instead of approximating the quadratures occurring in (2.2) by mechanical formulas, the unknown solution $y(x)$ itself is replaced by an approximating function $Y(x)$. The approximating function $Y(x)$ is defined in terms of $N+2$ parameters $y_{j}$ in a manner such that $Y(x)$ assumes the value $y_{j}$ at the mesh point $x_{j}$, i.e., $Y\left(x_{j}\right)=y_{j}$ $(j=0, \cdots, N+1)$. More specifically, in each interval $x_{i} \leq x<x_{i+1}$ the approximating function $Y(x)$ is defined to be a polynomial $Y_{i}(x)$ of degree $m$ passing through $y_{i}$ and $m$ other prescribed nearby ordinates. The boundary conditions (1.2) determine two of the ordinates in terms of the others. Then the integral (cf., equation (2.2))

$$
I(Y)=\int_{a}^{b}\left(Y^{\prime 2}-q Y^{2}-\lambda r Y^{2}\right) d x
$$

is a function of the values $y_{j}$ assumed by $Y(x)$ at $N$ distinct selected points. Requiring that the approximating function $Y(x)$ be an extremal of (7.1) leads, without further approximations, to an algebraic characteristic value problem determining $N$ sets of values for $\lambda$ and the ordinates $y_{j}$.

The polynomials $Y_{i}(x)$ defining the function $Y(x)$ are given by the Lagrange interpolation formula

$$
\begin{aligned}
Y_{i}(x)=\frac{\left(x-x_{i_{1}}\right) \cdots\left(x-x_{i_{m}}\right)}{\left(x_{i_{0}}-x_{i_{1}}\right) \cdots\left(x_{i_{0}}-x_{i_{m}}\right)} y_{i_{0}} & +\cdots \\
& +\frac{\left(x-x_{i_{0}}\right) \cdots\left(x-x_{i_{m-1}}\right)}{\left(x_{i_{m}}-x_{i_{0}}\right) \cdots\left(x_{i_{m}}-x_{i_{m-1}}\right)} y_{i_{m}}
\end{aligned}
$$


where $i_{k}=i+k-l_{i}(k=0,1, \cdots, m)$ and the $l_{i}$ are chosen such that $Y(x)$ is given by "forward" or "backward" interpolation formulas near the end points and by nearly "central" formulas at interior points and such that the interpolation polynomials are chosen symmetrically with respect to the midpoint $(a+b) / 2$. More specifiçally, in obtaining the numerical results reported in this paper $N$ is odd and the choice of the ordinates through which each $Y_{i}(x)$ is required to pass is equivalent to the following rules determining the single integer $l_{i}$

$$
\begin{array}{ll}
\quad l_{i}=i & \text { for } i \leq \frac{1}{2} m-1 \\
\frac{1}{2} m-2<l_{i} \leq \frac{1}{2} m-1 & \text { for } \frac{1}{2} m-1<i<\frac{1}{2} N \\
\frac{1}{2} m \leq l_{i}<\frac{1}{2} m+1 & \text { for } \frac{1}{2} N<i<N+1-\frac{1}{2} m \\
l_{i}=i+m-N-1 & \text { for } i \geq N+1-\frac{1}{2} m .
\end{array}
$$

Henceforth we shall assume that the mesh points $x_{i}$ are equally spaced with $x_{0}=a, x_{N+1}=b$ and $x_{i+1}-x_{i}=h=(b-a) /(N+1)$.

Since each $Y_{i}(x)$ depends linearly upon the ordinates $y_{j}$, its derivatives do also. Hence we may write for the $r$ th derivative of $Y_{i}(x)$ at $x_{i}$

$$
\frac{h^{r}}{r !} Y_{i}^{(r)}\left(x_{i}\right)=\sum_{j=1}^{N} \alpha_{r j}^{i} y_{j}
$$

For fixed values of $i$ and $r$, at most $m+1$ of the coefficients $\alpha^{i}{ }_{r j}$ are different from zero. The summation in (7.4) extends from $j=1$ to $j=N$ since $y_{0}$ and $y_{N+1}$ may be determined in terms of the others by the boundary conditions (1.2). The $\alpha_{r j}^{i}$ thus will in general depend upon the boundary conditions. In the special case where $\alpha_{2}=\beta_{2}=0$, however, we have $y_{0}=y_{N+1}=0$, and the $\alpha^{i}{ }_{r j}$ are rational numbers which can be expressed as integers divided by $m$ ! From (7.4) it follows by Taylor's theorem that

$$
Y_{i}(x)=\sum_{r=0}^{m} \sum_{j=1}^{N} h^{-r} \alpha^{i}{ }_{r j} y_{j}\left(x-x_{i}\right)^{r} .
$$

We shall discuss the determination of the quantities $\alpha^{i}{ }_{r j}$ in the next section. In the remainder of this section we assume that they are known and evaluate equation (7.1) for

$$
Y(x)=Y_{i}(x) \quad x_{i} \leq x<x_{i+1}
$$

where $Y_{i}(x)$ is given by equation (7.5).

Substituting equation (7.6) into (7.1) gives

$$
\begin{aligned}
I(Y)=\sum_{i=0}^{N} \int_{x_{i}}^{x_{i+1}}\left\{\left[\sum_{r} \sum_{j} r h^{-r} \alpha^{i}{ }_{r j} y_{j}\left(x-x_{i}\right)^{r-1}\right]^{2}\right. \\
\left.-[q(x)+\lambda r(x)]\left[\sum_{r} \sum_{j} h^{-r} \alpha^{i}{ }_{r j} y_{j}\left(x-x_{i}\right)^{r}\right]^{2}\right\} d x .
\end{aligned}
$$


If we define

$$
\begin{aligned}
P_{r t}^{i} & =\frac{r t}{h^{r+t+1}} \int_{x_{i}}^{x_{i+1}}\left(x-x_{i}\right)^{r+t-2} d x, \\
Q^{i}{ }_{r t} & =\frac{1}{h^{r+t+1}} \int_{x_{i}}^{x_{i+1}} q(x)\left(x-x_{i}\right)^{r+t} d x, \\
R_{r t}^{i} & =\frac{1}{h^{r+t+1}} \int_{x_{i}}^{x_{i+1}} r(x)\left(x-x_{i}\right)^{r+t} d x, \\
L_{j k} & =\sum_{i=0}^{N} \sum_{r, t=0}^{m} \alpha_{r j}^{i}\left(P_{r t}^{i}-Q_{r t}^{i}\right) \alpha^{i}{ }_{t j}, \\
D_{j k} & =\sum_{i=0}^{N} \sum_{r, t=0}^{m} \alpha_{r j}^{i} R_{r t}^{i} \alpha_{t k}^{i},
\end{aligned}
$$

equation (7.7) becomes

$$
I(Y)=\sum_{j, k=0}^{N}\left(L_{j k}-\lambda D_{j k}\right) y_{j} y_{k} .
$$

The extrema of $I(Y)$ are determined by the numbers $y_{j}$ satisfying

$$
\sum_{k=1}^{N} L_{j k} y_{k}=\lambda \sum_{k=1}^{N} D_{j k} y_{k} .
$$

for $j=1, \cdots, N$. This equation may be written in matrix notation as

$$
L Y=\lambda D Y
$$

when $Y$ is the matrix of a single column:

$$
Y=\left[\begin{array}{l}
y_{1} \\
\cdot \\
\cdot \\
\cdot \\
y_{N}
\end{array}\right] .
$$

8. Determination of $\alpha^{i}{ }_{r j}$. Formulas for the determination of the numbers $\alpha^{i}{ }_{r j}$ may be obtained by differentiation of equation (7.2) and using the boundary conditions. However, as will be shown below we may also determine these quantities by a double induction, one on the index $i$ and one on the degree of the polynomials involved. For simplicity in the ensuing discussion we assume that we are dealing with the special boundary conditions

$$
y_{0}=y_{N+1}=0 \text {. }
$$

Similar methods apply in the case of general boundary conditions. 
We first discuss the inductive procedure we use to determine the $\alpha^{0}{ }_{r j}$ which are defined by the equation

$$
\frac{h^{r} Y_{0}^{(r)}\left(x_{0}\right)}{r !}=\sum_{j=0}^{N+1} \alpha_{r j}^{0} y_{j}
$$

where $Y_{0}(r)\left(x_{0}\right)$ is the $r$ th derivative of the polynomial $Y_{0}(x)$ evaluated at $x=x_{0}$. By definition $Y_{0}(x)$ is the $m$ th degree Lagrange polynomial passing through $y_{0}, y_{1}, \cdots, y_{m}$. Then

Let $P_{s}(x)$ be the sth degree Lagrange polynomial passing through $y_{0}, y_{1}, \cdots, y_{s}$.

We note that

$$
Y_{0}(x)=P_{m}(x)
$$

$$
P_{0}(x) \equiv y_{0}
$$

In general we have

$$
P_{s}(x)=\sum_{k=0}^{s} p_{s k}(x) y_{k}
$$

where

$$
p_{s k}(x)=\frac{\left(x-x_{0}\right) \cdots\left(x-x_{k-1}\right)\left(x-x_{k+1}\right) \cdots\left(x-x_{s}\right)}{\left(x_{k}-x_{0}\right) \cdots\left(x_{k}-x_{k-1}\right)\left(x_{k}-x_{k+1}\right) \cdots\left(x_{k}-x_{s}\right)}
$$

We may also write

$$
P_{s}(x)=\sum_{r=0}^{s} \frac{P_{s}^{(r)}\left(x_{0}\right)}{r !}\left(x-x_{0}\right)^{r}
$$

where $P_{s}(r)\left(x_{0}\right)$ is the $r$ th derivative of $P_{s}(x)$ evaluated at $x=x_{0}$. We may further write

$$
\frac{h^{r}}{r !} P_{s}^{(r)}\left(x_{0}\right)=\sum_{k=0}^{N+1} \beta_{r k}^{s} y_{k}
$$

Then

$$
\alpha_{r j}^{0}=\beta^{m}{ }_{r j}
$$

and

$$
\beta_{r j}^{s}=\frac{h^{r}}{r !} p_{s j}^{(r)}\left(x_{0}\right)
$$

It follows from equations (8.4) and (8.5) that

$$
p_{s+1 k}=\frac{x-x_{s+1}}{x_{k}-x_{s+1}} p_{a k} \quad k<s+1
$$


(8.10)

$$
\begin{aligned}
p_{s+1 s+1} & =\frac{\left(x-x_{s}\right)}{\left(x_{s+1}-x_{s}\right)} \frac{\left(x_{s}-x_{0}\right)\left(x_{s}-x_{1}\right) \cdots\left(x_{s}-x_{s-1}\right)}{\left(x_{s+1}-x_{0}\right)\left(x_{s+1}-x_{1}\right) \cdots\left(x_{s+1}-x_{s-1}\right)} p_{s s}(x) \\
& =\frac{\left(x-x_{s}\right)}{h(s+1)} p_{s s}(x)
\end{aligned}
$$

the last equation holding when the intervals are equal in length.

Differentiating equations (8.9) and (8.10) $r$ times we have

$$
\begin{aligned}
p_{s+1}^{(r)}(x) & =\frac{x-x_{s+1}}{x_{k}-x_{s+1}} p_{s k}^{(r)}(x)+\frac{r}{x_{k}-x_{s+1}} p_{s k}^{(r-1)}(x) \\
p_{s+1}^{(r)}{ }_{s+1}(x) & =\frac{x-x_{s}}{h(s+1)} p_{s s}^{(r)}(x)+\frac{r}{h(s+1)} p_{s s}^{(r-1)}(x) .
\end{aligned}
$$

In view of equation (8.8) these equations may be written as

$$
\begin{aligned}
& \beta_{r k}^{s+1}=\frac{s+1}{s+1-k} \beta_{r k}^{s}-\frac{1}{s+1-k} \beta_{r-1 k}^{s} \\
& \beta_{r s+1}^{s+1}=-\frac{s}{s+1} \beta_{r s}^{s}+\frac{1}{s+1} \beta_{r-1 s}^{s} .
\end{aligned}
$$

It follows from equation (8.3) that

$$
\begin{aligned}
& \beta_{00}^{0}=1 \\
& \beta_{r k}^{0}=0 \quad r \neq 0, k \neq 0 .
\end{aligned}
$$

These equations together with equations (8.11) define $\beta_{r j}^{s}$ and hence $\alpha_{r j}^{0}$ in view of equation (8.7).

To obtain formulas by which the $\alpha_{r j}^{i}$ may be computed for $i>0$ we consider first the case in which $Y_{i}(x)$ and $Y_{i+1}(x)$ are the same polynomial. The $\alpha_{r j}^{i}$ and the $\alpha_{r j}^{i+1}$ then determine the derivatives of this polynomial at $x_{i}$ and $x_{i+1}$ respectively. Since $Y_{i}(x)=Y_{i+1}(x)$ we have in particular

$$
\frac{h^{r}}{r !} Y_{i}^{(r)}\left(x_{i+1}\right)=\frac{h^{r}}{r !} Y_{i+1}^{(r)}\left(x_{i+1}\right)
$$

Now

$$
\begin{aligned}
Y_{i}^{(r)}\left(x_{i+1}\right) & =\sum_{t=r}^{m} Y_{i}^{(t)}\left(x_{i}\right) \frac{h^{t-r}}{(t-r) !} \\
\frac{h^{r}}{r !} Y_{i}^{(r)}\left(x_{i+1}\right) & =\sum_{t=r}^{m} \frac{t !}{r !(t-r) !} \frac{h^{t}}{t !} Y_{i}^{(t)}\left(x_{i}\right) \\
& =\sum_{t=r}^{m} \frac{t !}{r !(t-r) !} \sum_{j=1}^{N} \alpha_{t j}^{i} y_{j} .
\end{aligned}
$$

Since $\frac{h^{r}}{r !} Y_{i+1}^{(r)}=\sum_{j=1}^{N} \alpha_{r j}^{i+1} y_{j}$ it follows that

$$
\alpha_{r j}^{i+1}=\sum_{t=r}^{m} \frac{t !}{r !(t-r) !} \alpha_{l j}^{i}
$$


If $N$ is chosen to be an odd integer and if the ordinates through which each $Y_{i}(x)$ is required to pass are chosen in accordance with (7.3) then (8.12) is applicable for $i<\frac{1}{2} m-2$ and for $i>N+1-\frac{1}{2} m$.

When the ordinates through which $Y_{i}(x)$ and $Y_{i+1}(x)$ are chosen in the same way, as for example when $Y_{i+1}(x)$ passes through $y_{i-1}, y_{i}$, and $y_{i+1}$ and $Y_{i+1}(x)$ passes through $y_{i}, y_{i+1}$, and $y_{i+2}$, then clearly

$$
\alpha_{r j}^{i+1}=\alpha_{r j-1}^{i} \text {. }
$$

This formula is applicable, in accordance with (7.3), except near the end points and at the midpoint $x_{M}$, where $M=\frac{1}{2}(N+1)$. If $m$ is even we find that $Y_{M}(x)$ $=Y_{M-1}(x)$ so that (8.12) applies. If $m$ is odd then it follows from (7.3) that $Y_{M}(x)=\cdot Y_{M-2}(x)$ so that the induction formula (8.12) must be used twice. Noting that $\alpha_{r j+1}^{M-1}=\alpha_{r j}^{M-2}$. we have

$$
\alpha_{r j}^{M}=\sum_{s=r}^{m} \frac{s !}{r !(s-r) !} \sum_{t=8}^{m} \frac{t !}{s !(t-s) !} \alpha_{t j+1}^{M-1}
$$

when $m$ is odd. Summarizing these results

$$
\begin{aligned}
& i \leq \frac{m}{2}-2 \\
& \alpha_{r j}^{i+1}=\sum_{t=r}^{m} \frac{t !}{r !(t-r) !} \alpha_{t j}^{i} \quad \text { for } \quad i \geq N+1-\frac{m}{2} \\
& i=M-1 \text {, if } m \text { is even } \\
& \alpha_{r j}^{M}=\sum_{s=r}^{m} \frac{s !}{r !(s-r) !} \sum_{t=s}^{m} \frac{t !}{s !(t-s) !} \alpha_{r j+1}^{M-1} \text { if } m \text { is odd } \\
& \frac{m}{2}-2<i<M-1 \\
& \alpha_{r j}^{i+1}=\alpha_{r j-1}^{i} \\
& \text { for } \\
& M-2<i<N+1-\frac{m}{2}
\end{aligned}
$$

where, again, $N$ is odd and $M=\frac{1}{2}(N+1)$. These equations together with our previous results for determining the $\alpha^{0}{ }_{r j}$ constitute a means for computing the coefficients $\alpha^{i}{ }_{r j}$ for $i=0, \cdots, N ; r=0, \cdots, m$; and $j=1, \cdots, N$ for the boundary conditions $y(a)=y(b)=0$ when $N$ is chosen to be odd.

9. The Computation Procedure. Both Method I and Method II lead to algebraic problems of the type given by equation (7.13). In the former case the matrix $D$ is diagonal and various simplifications occur. For both methods the computation proceeds in two similar steps: (1) computing the elements of the matrices $L$ and $D$, and (2) solving equations (7.13) for $\lambda$ and the $y_{j}$.

In Method I the matrix $L$ is determined from the matrices $a_{i j}$ given by equations (5.3) or (6.3) in accordance with equation (3.4).

In Method II step (1) begins with the computation of $\alpha_{r j}^{0}$. We have initially a matrix with $m+1$ columns, $r=0, \cdots, m$ and $N+1$ rows, $j=0, \cdots, N$, 
only the element $(0,0)$ being different from 0 . Then the transpose of the matrix $\left\|\alpha_{r}{ }_{r j}\right\|$ is computed using the formulas derived in section 8 . Thereafter the first row is no longer needed (assuming boundary conditions $y(a)=y(b)=0$ ). Then we have routines which perform the following functions: (1) compute $\left\|\alpha^{i}{ }_{r j}\right\|$ from $\left\|\alpha_{r j}^{i-1}\right\|$ using equations (8.13); (2) compute the matrix $\left\|P^{i}-Q^{i}\right\|$ using equations (7.8) and (7.9); (3) matrix multiplication $\sum_{r=0}^{m} \alpha_{r t}^{i}\left(P_{r t}^{i}-Q_{r t}^{i}\right)$; (4) matrix multiplication $\sum_{i=0}^{m} \alpha_{t k}^{i}\left[\sum_{r=0}^{m} \alpha_{r j}\left(P_{r t}^{i}-Q_{r t}^{i}\right)\right]$ and addition of the result to the previous partial sum; (5) compute the matrix $\left\|R^{i}\right\|$ using equations (7.10); (6) (master routine) step $i$ and repeat until $i=N+1$. Routines (3) and (4) are used to form the partial sums for both $L$ and $D$. Likewise (3) and (5) may conveniently be combined into a single routine. Since $\left\|P^{i}-Q^{i}\right\|,\left\|R^{i}\right\|$ are both symmetric and can use the same storage locations, since $L$ and $D$ are symmetric, and since $\left\|\alpha^{i}\right\|^{T} \cdot\left\|P^{i}-Q^{i}\right\|$ and $\left\|\alpha^{i}\right\|^{T} \cdot\left\|R^{i}\right\|$ can use the same storage locations, a total of $\frac{1}{2}(m+1)(m+2)+N(N+1)+2 N(m+1)$ matrix storage locations are required. It is also time-saving to store the integers 0 ! through $m$ ! for use in computing the $\left\|\alpha^{i}\right\|$.

If the functions $q(x)$ and $r(x)$ are symmetric with respect to $x=\frac{1}{2}(a+b)$ it is time-saving to choose $N$ to be odd and carry out the "integration" only from $x_{M}=\frac{1}{2}(a+b)$ to $x_{N+1}=b$. Then the correct result is obtained by replacing the elements $L_{j k}\left(D_{j k}\right)$ and $L_{N-j+1, N-k+1}\left(D_{N-j+1, N-k+1}\right)$ both by their sum for $j, k \leq M$. In obtaining the numerical results reported in this paper this procedure was followed. The matrix $\alpha^{M}{ }_{r j}$ was computed utilizing a numerical differentiating routine which uses polynomial approximations, but a procedure similar to that outlined for obtaining $\alpha^{0}{ }_{r j}$ could have been followed.

In both methods, the scheme used in step (2) for solving the equation

$$
L y=\lambda D y
$$

begins with the computation of the characteristic values and characteristic vectors of the matrix $D$, to obtain the decomposition $D=U^{T} \Delta U$, where $\Delta$ is a diagonal matrix containing the characteristic values of $D$ and $U$ is a unitary matrix containing its characteristic vectors. Then

$$
\left[\left(\Delta^{-1} U^{T}\right)^{T} L\left(\Delta^{-1} U^{T}\right)\right]\left(U \Delta^{\frac{1}{2}} Y\right)=\lambda\left(U \Delta^{1} Y\right) .
$$

The matrix shown in brackets is computed and then diagonalized to obtain the characteristic values $\lambda$ and the vectors $U \Delta^{i} Y$. The characteristic vectors $Y=\Delta^{-\frac{1}{2}} U^{T}\left(U \Delta^{\frac{1}{3}} Y\right)$ are then computed. Both diagonalizations are done by the Jacobi method (see Gregory [3]). However for Method I advantage is taken of the fact that $D$ is already a diagonal matrix.

Since, except for the factor $r t$ in the definition of $P^{i_{r t}}$, the elements $P^{i}{ }_{r t}, Q^{i}{ }_{r t}$, $R_{r t}^{i}$ depend only on the sum $r+t$ and $i$, a total of $(N+1)(6 m+2)$ quadratures, each over an interval of length $h$, are required. Computing the matrices $L$ and $D$ requires a total of $m(N+1)\left(N^{2}+N+2 m\right)$ multiplications. The most troublesome term in this expression is $m N^{3}$; this is to be compared with $50 N^{3}$, which is approximately the number of multiplications required in the two diagonalizations. Thus the time required to compute the elements of $L$ and $D$ is small in comparison to the time required to solve the equation $L y=\lambda D y$. 
10. Numerical Results and Their Discussion. In this section we shall report and discuss the results obtained by applying Methods I and II to the example given by equations (6.7) to (6.9). The results are given in tabular form. Each entry represents the value of $100\left(\lambda_{k}{ }^{*}-\lambda_{k}\right) / \lambda_{k}$ where $\lambda_{k}$ is the characteristic value as computed from equation (6.10) with $n=k$ and $\lambda_{k}{ }^{*}$ is the $k$ th approximate characteristic value obtained by solving the equation

$$
L y=\lambda D y \text {. }
$$

Results on the comparison of the $y_{i}$ which satisfy the above equation and $y\left(x_{i}\right)$ computed from equation (6.11) are also available but not reported herein because of space limitations.

TABLE 1. $100\left(\lambda_{k}^{*}-\lambda_{k}\right) / \lambda_{k}$ from Method I with $N=19$

$\begin{array}{lccccccccc}\quad k= & 1 & 2 & 3 & 4 & 5 & 6 & 7 & 8 & 9 \\ \text { 1st Order } & 4.97 & 4.29 & 3.16 & 1.60 & -.37 & -2.75 & -5.49 & -8.59 & -11.99 \\ \text { 2nd Order } & .00 & -.03^{*} & -.15 & -.47^{*} & -1.17 & -2.46^{*} & -4.60 & -7.89 & -12.60 \\ \text { 2nd Order* } & .00 & .00^{*} & -.14 & -.47^{*} & -1.17 & -2.45^{*} & -4.60 & -7.88 & -12.59\end{array}$

Not all the computed $\lambda^{* \prime}$ s are reported in these tables. In case $N=19$ we give nine $\lambda^{*}$ and in case $N=9$ we give five $\lambda^{*}$. The reason for this is that the true characteristic values $\lambda_{n}$ for $n \geq 10$ when $N=19$ and $n \geq 5$ when $N=9$ correspond to functions which have a maximum within the first interval used. Hence any approximation of this function by the value of its ordinate at the end of the interval will not be a reasonable one and would be expected to lead to poor results, as was found to be the case.

In Table 1 which contains the results obtained by the use of Method I with $N=19$ still an additional selection principle was used in choosing the value of $\lambda_{k}{ }^{*}$ reported. The first nine values of $\lambda^{*}$ which were associated with values of $y_{i}{ }^{*}$,

TABLE 2. $100\left(\lambda_{k}{ }^{*}-\lambda_{k}\right) / \lambda_{k}$ from Method II with $N=9$

$\begin{array}{cccccc}m / k & 1 & 2 & 3 & 4 & 5 \\ 2 & .018 & .309 & 1.249 & 4.192 & 7.498 \\ 3 & .001 & .052 & .645 & 2.224 & 7.299 \\ 4 & .000 & .015 & .022 & .323 & 1.945 \\ 5 & .000 & .000 & .019 & .104 & .767 \\ 6 & .000 & .000 & .003 & .106 & .383 \\ 7 & .000 & .000 & .000 & .028 & .723 \\ 8 & .000 & .000 & .000 & .004 & .203\end{array}$

such that a continuous function passing through these ordinates would have the same number of zeros as the corresponding solution of the Sturm-Liouville equation, were used. That is, the known oscillation properties of solutions of Sturm-Liouville systems were used to distinguish between "true" approximations to $\lambda_{k}$ and "spurious" ones when two close values of $\lambda_{k}{ }^{*}$ were obtained. The entries marked with an asterisk in Table 1 were one of a pair of computed values of $\lambda^{*}$ which were numerically close.

The first line of Table 1 contains the results obtained by using the first order expressions for $a_{i j}$ and $w_{i}$ (equations (5.3) and (5.4) in equation (3.4)). The second line was obtained by using the second order expressions for $a_{i j}$ and $w_{i}$ (equations 
(6.3) and (6.4)). The third line was obtained by modifying the even rows in the body of the matrix in equation (6.3) so that they are of the form

instead of

$$
2^{-15}-\left(\frac{1}{2}+2^{-14}\right) \quad 0 \quad\left(\frac{1}{2}+2^{-14}\right) \quad-2^{-15}
$$

$$
\begin{array}{lllll}
0 & -\frac{1}{2} & 0 & \frac{1}{2} & 0 .
\end{array}
$$

The fact that the entries of the third row are uniformly smaller than those of the second shows that there is no advantage to be obtained in Method I by requiring the $a_{i j}$ and $w_{j}$ to be chosen so that the resulting algebraic equation is a second order approximation to the original differential equation. Indeed this example shows that by modifying the $a_{i j}$ and $w_{j}$ satisfying this condition, so that the condition is violated, better results can be obtained.

Table 2 gives the results obtained by using Method II. The entries in a single row correspond to a fixed value of $m$ labelling that row. As was pointed out earlier, the total computation time is not seriously changed as $m$ is increased from 2 to 8 . By using $m=8$ one can obtain all the characteristic values one has a right to expect to get with an accuracy less than or equal to .203 per cent.

\begin{tabular}{cccccccccc}
\multicolumn{8}{c}{ TABLE 3. $100\left(\lambda_{k}^{*}-\lambda_{k}\right) / \lambda_{k}$ from Modified Method II (with $\left.m=2\right)$} \\
$k=$ & 1 & 2 & 3 & 4 & 5 & 6 & 7 & 8 & 9 \\
$N=19$ & .00 & -.01 & -.05 & -.13 & -.32 & -.67 & -1.39 & -3.09 & -7.53 \\
$N=9$ & -.010 & -.137 & -.655 & -3.075 & -18.943 & & & & \\
$N=9$ & .011 & .126 & .389 & .674 & 1.34 & & & & \\
$N=9$ & .018 & .308 & 1.249 & 4.192 & 7.498 & & & &
\end{tabular}

The first two rows in Table 3 contain results obtained by using a modification of Method II. In the first row of this table $N=19$ and in the last three rows $N=9$. The last row of Table 3 is the first row of Table 2. The modifications referred to are the following: The polynomials $Y_{i}(x)$ used to replace the functions $y(x)$ in the integral (2.2) are such that

$$
\begin{aligned}
Y_{0}(x) & =P_{2}(x) \\
Y_{i+1}\left(x_{i+1}\right) & =Y_{i}\left(x_{i+1}\right) \\
Y_{i+1}^{\prime}\left(x_{i+1}\right) & =Y_{i}^{\prime}\left(x_{i+1}\right) .
\end{aligned}
$$

Further, for the first two rows of Table 3 the matrix $D$ is made diagonal by evaluating

$$
\int_{a}^{b} r y^{2} d x
$$

by means of Simpson's rule instead of replacing $y(x)$ by $Y(x)$ defined above.

This last approximation procedure means that the computation time required for solving the equation

$$
L y=\lambda D y
$$

is markedly decreased. However, it is no longer true that the $\lambda_{k}{ }^{*}$ are always greater than the $\lambda_{k}$. 
On comparing the second and fourth rows of Table 3 we see that for the first four characteristic values the results obtained from the modified Method II are better than those given by the unmodified one for $N=9$. Other computations have shown that this result does not hold for $m \neq 2$ if the class of admissible function is defined so as to have continuous derivatives at the points $x=x_{i}$ and if the matrix $D$ is evaluated as in Method II.

In the third row of Table 3 the matrix $D$ is evaluated as in Method II where the admissible functions are defined as for the first two rows.

The numerical results, although obtained only for a particular simple example, would seem to justify the use of the methods discussed above for the solution of more complicated Sturm-Liouville problems. They should be supplemented by a test for the accuracy of the results obtained for the characteristic values and characteristic functions applicable to problems for which the solution is not known. Various such tests are under investigation and will be reported on subsequently.

Digital Computer Laboratory

C. C. Farrington

University of Illinois

Urbana, Illinois

University of California

R. T. GREGORY

Goleta, California

Digital Computer Laboratory

A. H. TAUB

University of Illinois

Urbana, Illinois

This work was supported in part by the National Science Foundation under Grant G-1221.

1. F. B. Hildebrand, Introduction to Numerical Analysis, McGraw-Hill Book Co., New York, 1956, p. 241.

2. R. T. GREGORY, "A numerical solution to a class of Sturm-Liouville systems," University of Illinois thesis, 1955 .

3. R. T. GREGORY, "Computing eigenvalues and eigenvectors of a symmetric matrix on the ILLIAC," $M T A C$, v. 7, 1953, p. 215-220.

\section{On the Numerical Evaluation of the Stokes' Stream Function}

1. Introduction. In the study of axially symmetric problems in fluid dynamics, the Stokes' stream function, that is, the function which satisfies

$$
u_{x x}-\frac{1}{y} u_{y}+u_{y y}=0, \quad y \neq 0,
$$

is of considerable interest. This function is constant on the streamlines.

The problem to be considered is a Dirichlet type problem. Let $G$ be a closed, bounded, simply connected plane region whose interior is denoted by $R$ and whose boundary curve is denoted by $S$. Let $G$ not contain any point where $y=0$. 